\title{
Geranylgeranyltransferase I as a target for anti-cancer drugs
}

\author{
Mark R. Philips ${ }^{1}$ and Adrienne D. Cox ${ }^{2}$ \\ ${ }^{1}$ Departments of Medicine, Cell Biology, and Pharmacology, New York University Cancer Institute, New York University School of Medicine, New York, \\ New York, USA. ${ }^{2}$ Departments of Radiation Oncology and Pharmacology, Lineberger Comprehensive Cancer Center, \\ University of North Carolina School of Medicine, Chapel Hill, North Carolina, USA.
}

\begin{abstract}
Posttranslational modification is critical for the function of the gene products of ras oncogenes, which are frequently mutated in cancer. Ras proteins are modified by farnesyltransferase (FTase), but many related small GTPases that also end in a CAAX motif (where $C$ is cysteine, $A$ is often an aliphatic amino acid, and $X$ is any amino acid) are modified by a closely related enzyme known as geranylgeranyltransferase type $I$ (GGTase-I). Accordingly, inhibitors for both of these enzymes have been developed, and those active against FTase are in clinical trials. In this issue of the JCI, Sjogren et al. report the development of a mouse strain homozygous for a conditional allele of the gene that encodes GGTase-I (see the related article beginning on page 1294). They found that ablation of the GGTase-I-encoding gene in cells destined to produce lung tumors driven by oncogenic K-Ras resulted in delayed onset and decreased severity of disease, validating in a genetic model the theory that GGTase-I is a good target for anti-cancer drug development.
\end{abstract}

Ras is mutated in human cancer more frequently than any other oncogene. Accordingly, Ras has been a center of focus for cancer biologists seeking to develop rationally designed anti-cancer drugs. In 1989, several groups showed that Ras proteins are posttranslationally modified by a 15-carbon farnesyl lipid (1) that was later shown to be essential for membrane association and transformation (2). Within a year, farnesyltransferase (FTase) was purified and characterized in the laboratory of Brown and Goldstein (3). One year later the same group showed that a second prenyltransferase, geranylgeranyltransferase type I (GGTase-I), modifies Ras-related GTPases with a 20-carbon geranylgeranyl lipid (4). GGTase-I was later shown to modify Ras when FTase was blocked (5). Hamilton and Sebti have pioneered the development of GGTase-I inhibitors (GGTIs) (6-8). Sjogren et al. now report in this issue of the JCI (9) the development

Nonstandard abbreviations used: $\mathrm{Cdc} 42$, cell division cycle 42; FTase, farnesyltransferase; FTI, FTase inhibitor; GGTase-I, geranylgeranyltransferase type I; GGTI, GGTase-I inhibitor; MEF, mouse embryonic fibroblast; Pggt $1 b$, gene encoding the $\beta$ subunit of GGTase-I.

Conflict of interest: The authors have declared that no conflict of interest exists.

Citation for this article: J. Clin. Invest. 117:1223-1225 (2007). doi:10.1172/32108. of a mouse that is conditionally deficient in GGTase-I.

\section{Prenyltransferases as drug targets}

Ras is member of a class of proteins that terminate in a CAAX sequence, so called for the invariant cysteine and the often, but not always, aliphatic ("A") residues. These proteins are subject to a series of posttranslational modifications of the CAAX sequence that include polyisoprenylation, proteolysis, and carboxyl methylation (Figure 1). The first and rate-limiting step in CAAX processing is catalyzed by one of the two prenyltransferases. FTase and GGTase-I are cytosolic heterodimers that share a common $\alpha$ subunit but use different $\beta$ subunits. A third prenyltransferase, GGTase-II, consists of distinct subunits and modifies Rab proteins. Because Ras proteins are substrates for FTase and inhibition of FTase prevents all subsequent modifications, this enzyme emerged as an obvious initial target for drug development.

Over the past 15 years, many pharmaceutical companies have developed FTase inhibitors (FTIs). The preclinical testing of these agents in cell culture and animal models of cancer was very impressive overall, and several of the compounds have reached phase III clinical trials (10). Despite the fact that multiple regulatory proteins, including elements of the visual signal transduction pathway, are substrates for FTase, FTIs are remarkably well tolerated. Unfortunately, the efficacy of these agents against tumors has been disappointing. Although some single-agent activity toward hematopoietic malignancies has been demonstrated (10), no such activity has been found for solid tumors. Ongoing trials are designed to test FTIs in combination with other chemotherapeutic agents (10).

Two major surprises have resulted from FTI research. First, the efficacy of these agents in preclinical testing did not correlate with the presence or absence of activating mutations of Ras (11), suggesting that FTIs have at least one other target through which they can limit cell proliferation and survival. This observation has initiated an ongoing quest to identify which of the more than 50 proteins encoded in the human genome that are predicted to be substrates for FTase (12) might be the relevant target(s). Second, although model tumors driven by oncogenic H-Ras were highly susceptible to FTIs, tumors driven by oncogenic N-Ras and K-Ras were not (10). This was particularly disappointing, as the vast majority of human solid tumors driven by oncogenic Ras involve $\mathrm{K}$-Ras and hematopoietic malignancies are driven by N-Ras. Part of the explanation for the resistance of K-Ras and N-Ras proved to be alternative prenylation, whereby in the presence of FTIs, N-Ras and K-Ras, but not H-Ras, become substrates for GGTase-I and retain full biological activity (5). The latter observation has helped to propel interest in GGTase-I as an additional therapeutic target.

Alternative prenylation of Ras is but one impetus for the development of GGTIs. Several geranylgeranylated CAAX proteins are critical for oncogenesis downstream of Ras (13-17). GGTIs have now been developed to target these substrates and, although the first clinical trials will not begin until later this year, some encouraging preclinical data have emerged. When 


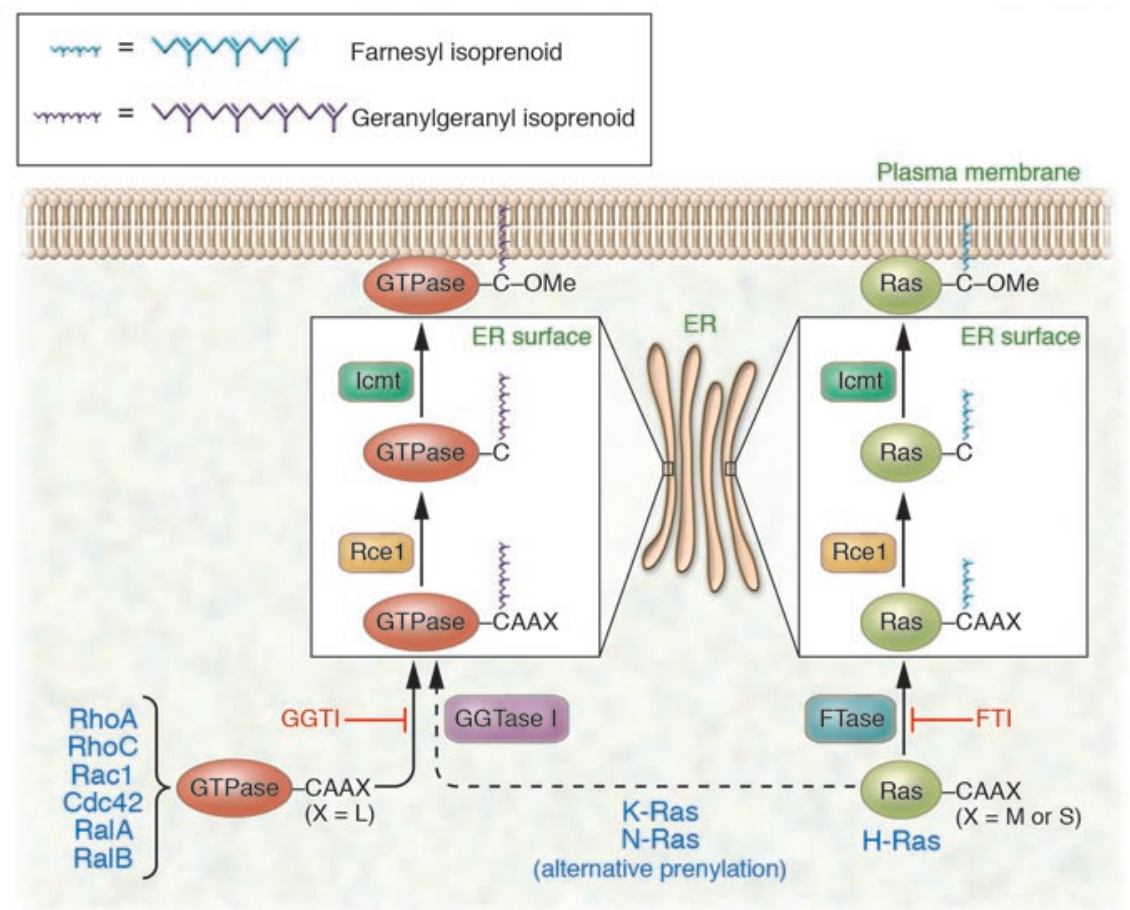

\section{Figure 1}

Prenylation of CAAX proteins. The CAAX sequence of Ras proteins ends in methionine $(\mathrm{M})$ or serine $(\mathrm{S})$ and is therefore recognized preferentially by FTase, which lipidates the protein with a 15-carbon farnesyl isoprenoid at the CAAX cysteine. Farnesylated Ras is then further processed on the surface of the ER by a protease (Rce1) and isoprenylcysteine carboxyl methyltransferase (Icmt) and then transported to the plasma membrane. The product of this three-step processing is a C-terminal cysteine that is both farnesylated and methyl esterified $(-\mathrm{OMe})$ such that it is hydrophobic and associates with the phospholipid bilayer. This entire processing sequence can be blocked by farnesyl transferase inhibitors (FTIs). Ras-related GTPases that end with a CAAX sequence in which X is a leucine (L) are lipidated instead with a 20-carbon geranylgeranyl isoprenoid by GGTase-I. Several of these proteins, e.g., RhoA, RhoC, Rac1, Cdc42, RalA, and RalB, are required for Ras-mediated cellular transformation, making GGTase-I a target for drug discovery. When FTase is inhibited by FTI, N-Ras and K-Ras are processed instead by GGTase-I, but $\mathrm{H}$-Ras cannot be alternatively prenylated. Alternative prenylation of Ras has heightened interest in the development of GGTIs. In this issue of the $\mathrm{JCl}$, Sjogren et al. (9) have conditionally deleted GGTase-I and shown that K-Ras-driven tumors are inhibited, presumably because one or more of the substrates for this enzyme are required for oncogenesis.

used alone, GGTase-I-selective inhibitors have shown the ability to impair transformation in vitro and tumor growth in vivo $(6-8,18)$. Unlike FTIs, GGTIs cause both cell cycle arrest in $\mathrm{G} 1$ and apoptosis $(6-8)$. The mechanistic bases for these effects are beginning to be elucidated. Interestingly, the severe toxicity once predicted for GGTIs, like that originally predicted for FTIs, is far less significant than supposed (8). However, the antineoplastic targets of GGTIs remain uncertain, and better preclinical models for evaluating the consequences of GGTase-I inhibition in vivo are clearly warranted.

\section{GGTase-I deficiency ameliorates K-Ras-driven tumors}

A genetic model of GGTase-I deficiency would certainly be helpful in further assessing the potential of GGTase-I as a target for anti-cancer drugs. In their report in this issue of the JCI, Sjogren and colleagues generated a conditional knockout allele for the $\beta$ subunit of GGTase-I (Pggt1b), in which exon 7 , critical for enzymatic activity, is flanked by loxP sites (9). The authors studied mouse embryonic fibroblasts (MEFs) from $P g g t 1 b^{f l / f l}$ mice in which both copies of the Pggt $1 b$ gene could be ablated by infection with an adenovirus directing expression of Cre recombinase. GGTase-I-deficient cells accumulated $p 21^{\text {CIP1/Waf1 }}$ and failed to proliferate, concordant with the effects of GGTIs (7). Nevertheless, GGTase-Ideficient cells remained viable for at least three weeks, demonstrating that GGTase-I is not required for basic metabolic activity. Not surprisingly, GGTase-I-deficient cells were small and spindle shaped with little polymerized actin and reduced motility.

Interestingly, although more than 50 proteins in the human genome are predicted to be substrates for GGTase-I and not FTase, partial rescue of cell proliferation could be accomplished by expressing farnesylated forms of just two of these, RhoA and cell division cycle 42 (Cdc42) (9). Does this mean that other substrates for GGTase-I previously thought to be critical for cell growth and survival, such as Rac and Ral proteins, are not in fact required? Are they required but act upstream of RhoA and Cdc42? Or do some GGTase substrates undergo alternative prenylation by FTase? Although the authors show that, in GGTase-I-deficient cells, a large amount of unprenylated Rap1 accumulates, this does not rule out the possibility of a farnesylated pool, and it will be important to explore this possibility further.

To study GGTase-I deficiency in vivo, Sjogren et al. (9) bred Pggt $1 b^{f l / f l}$ mice with mice carrying a conditional allele of oncogenic $\mathrm{K}$-Ras that is activated by Cre recombinase and with a strain of mice that expresses 
Cre driven by a myeloid-specific lysozyme $\mathrm{M}$ promoter. They then crossed the two compound transgenic strains. The authors hoped to create K-Ras-dependent myeloid malignancies in these mice with or without concomitant GGTase-I deficiency. The first surprise came from the observation that hematopoiesis was normal in mice selectively deficient in GGTase-I in myeloid cells. PCR analysis revealed a Cre recombination efficiency of $90 \%$, strongly suggesting that GGTase-I is not required for the development of myeloid cells. Functional analysis of these myeloid cells, particularly with regard to motility and NADPH oxidase activity, processes that require geranylgeranylated Rac2, will be important to assess in future studies.

The second surprise was that, rather than myeloid malignancies, the mice with activated K-Ras succumbed to rapidly progressive lung tumors (9). A likely explanation is that activated K-Ras was expressed in the lung because lysozyme $\mathrm{M}$ is expressed not only in myeloid cells but also in type II pneumocytes. Most important, GGTase-I deficiency dramatically extended the lifespan of mice expressing activated K-Ras by delaying the onset and lowering the severity of the lung tumors. Another phenomenon observed was a myeloid cell infiltration of the liver in mice with oncogenic K-Ras, a phenotype that was completely reversed in the setting of GGTase-I deficiency. Thus, two in vivo phenotypes driven by oncogenic K-Ras were ameliorated by GGTase-I deficiency. Interestingly, some lung tumors were found to have undergone Cre-mediated excision of both Pggt $1 b^{f l}$ alleles, demonstrating that, in some contexts, cells can proliferate without geranylgeranylated CAAX proteins.

How did GGTase-I deficiency limit the oncogenicity of K-Ras in the model reported by Sjogren et al. (9)? There is no reason to suspect that prenylation of K-Ras itself was affected, since FTase was fully active in the mouse tumors. The simplest explanation is the loss of function of one or more CAAX proteins that require geranylgeranylation for activity and that are required for the full expression of K-Ras-mediated oncogenesis. Logical candidates include Ral proteins (13, 14), RhoA (15), Rac1 $(15,19,20)$, Cdc42 (17), and RhoC (16).

\section{Unanswered questions, future studies}

The results reported by Sjogren et al. (9) further validate the idea of targeting GGTase-I for anti-cancer drug discovery. However, like all provocative studies, this one leaves us with many unanswered questions. Does the ability to rescue GGTase-I-deficient cell proliferation with few farnesylated substrates mean that, contrary to previous expectations, many GGTase-I substrates can be alternatively prenylated by FTase in the setting of GGTase-I deficiency? Are the antitumor effects of GGTase-I deficiency specific to Ras-dependent tumors? What are the geranylgeranylated proteins required to maximize $\mathrm{K}$-Ras oncogenesis in this model? Since FTIs have already been tested in patients and use of GGTIs are on the horizon, perhaps the most practical question that can be addressed with this new mouse model is whether FTIs will demonstrate more potent anti-Ras activity in the setting of GGTase-I deficiency and whether inhibiting both CAAX prenyltransferases can be accomplished without unacceptable in vivo toxicity.

Most important is the question of whether the results reported with this new K-Ras-driven lung tumor model will translate to human cancer. However, even if the model presented in this first report does not offer insight into human cancer, the Pggt $1 b^{f l / f l}$ mouse can be utilized by simple breeding with other mouse models of cancer in strains where Cre is expressed in a tissue-specific manner. In this way the Pggt $1 b^{f l / f l}$ mouse is likely to be an invaluable tool in assessing the role of GGTase-I in oncogenesis.

\section{Acknowledgments}

The authors thank Channing Der for helpful discussions.

Address correspondence to: Mark R. Philips, New York University School of Medicine, 522 First Avenue, New York, New York 10016, USA. Phone: (212) 263-7404; Fax: (212) 263-9210; E-mail: philim01@ med.nyu.edu.

\footnotetext{
1. Casey, P.J., Solski, P.A., Der, C.J., and Buss, J.E. 1989. p21ras is modified by a farnesyl isoprenoid. Proc. Natl. Acad. Sci. U. S. A. 86:8323-8327.
}

2. Jackson, J.H., et al. 1990. Farnesol modification of Kirsten-ras exon 4B protein is essential for transformation. Proc. Natl. Acad. Sci. U. S. A. 87:3042-3046.

3. Reiss, Y., Goldstein, J.L., Seabra, M.C., Casey, P.J., and Brown, M.S. 1990. Inhibition of purified $221^{\text {ras }}$ farnesyl:protein transferase by Cys-AAX tetrapeptides. Cell. 62:81-88.

4. Seabra, M.C., Reiss, Y., Casey, P.J., Brown, M.S., and Goldstein, J.L. 1991. Protein farnesyltransferase and geranylgeranyltransferase share a common alpha subunit. Cell. 65:429-434.

5. Whyte, D.B., et al. 1997. K- and N-Ras are geranylgeranylated in cells treated with farnesyl protein transferase inhibitors. J. Biol. Chem. 272:14459-14464.

6. Sun, J., et al. 1999. The geranylgeranyltransferase I inhibitor GGTI-298 induces hypophosphorylation of retinoblastoma and partner switching of cyclindependent kinase inhibitors. A potential mechanism for GGTI-298 antitumor activity. J. Biol. Chem. 274:6930-6934

7. Vogt, A., Sun, J., Qian, Y., Hamilton, A.D., and Sebti, S.M. 1997. The geranylgeranyltransferase-I inhibitor GGTI-298 arrests human tumor cells in G0/G1 and induces $\mathrm{p} 21$ (WAF1/CIP1/SDI) in a p53-independent manner. J. Biol. Chem. 272:27224-27229.

8. Sun, J., et al. 2003. Geranylgeranyltransferase I inhibitor GGTI-2154 induces breast carcinoma apoptosis and tumor regression in H-Ras transgenic mice. Cancer Res. 63:8922-8929.

9. Sjogren, A.-K.M., et al. 2007. GGTase-I deficiency reduces tumor formation and improves survival in mice with K-RAS-induced lung cancer. J. Clin. Invest. 117:1294-1304. doi:10.1172/30868.

10. Basso, A.D., Kirschmeier, P., and Bishop, W.R. 2006. Lipid posttranslational modifications: farnesyl transferase inhibitors. J. Lipid Res. 47:15-31.

11. Sepp-Lorenzino, L., et al. 1995. A peptidomimetic inhibitor of farnesyl:protein transferase blocks the anchorage-dependent and -independent growth of human tumor cell lines. Cancer Res. 55:5302-5309.

12. Reid, T.S., Terry, K.L., Casey, P.J., and Beese, L.S. 2004. Crystallographic analysis of CaaX prenyltransferases complexed with substrates defines rules of protein substrate selectivity. J. Mol. Biol. 343:417-433.

13. Hamad, N.M., et al. 2002. Distinct requirements for Ras oncogenesis in human versus mouse cells. Genes Dev. 16:2045-2057.

14. Chien, Y., and White, M.A. 2003. RAL GTPases are linchpin modulators of human tumour-cell proliferation and survival. EMBO Rep. 4:800-806.

15. Khosravi-Far, R., Solski, P.A., Clark, G.J., Kinch, M.S., and Der, C.J. 1995. Activation of Rac1, RhoA, and mitogen-activated protein kinases is required for Ras transformation. Mol. Cell. Biol. 15:6443-6453.

16. Clark, E.A., Golub, T.R., Lander, E.S., and Hynes, R.O. 2000. Genomic analysis of metastasis reveals an essential role for RhoC. Nature. 406:532-535.

17. Qiu, R.G., Abo, A., McCormick, F., and Symons, M. 1997. Cdc42 regulates anchorage-independent growth and is necessary for Ras transformation. Mol. Cell. Biol. 17:3449-3458.

18. Peterson, Y.K., Kelly, P., Weinbaum, C.A., and Casey, P.J. 2006. A novel protein geranylgeranyltransferase-I inhibitor with high potency, selectivity, and cellular activity. J. Biol. Chem. 281:12445-12450.

19. Qiu, R.G., Chen, J., Kirn, D., McCormick, F., and Symons, M. 1995. An essential role for Rac in Ras transformation. Nature. 374:457-459.

20. Joyce, P.L., and Cox, A.D. 2003. Rac1 and Rac3 are targets for geranylgeranyltransferase I inhibitormediated inhibition of signaling, transformation, and membrane ruffling. Cancer Res. 63:7959-7967. 